\title{
A NOTE ON JENSEN'S COVERING LEMMA
}

\author{
P. KOMJÁTH
}

ABstract. We show that Jensen's covering lemma does not hold for order-types instead of cardinalities.

A famous result of Jensen says that if $0^{\#}$ does not exist then every uncountable set can be covered by a constructible set of the same power [1]. We show that an iterated forcing construction can produce a model such that for every $\alpha<\omega_{2}$ there is a set (in the enlarged model) of order-type $\omega_{1}$ which cannot be covered by an old set of order-type $\leqslant \alpha$.

THEÓREM. Assume that $V$ is a countable, transitive model of $Z F C+G C H$. Then there is a cardinal preserving generic extension $V[G]$ such that for every $\alpha<\omega_{2}$ there is an $X \subseteq \omega_{2}, \operatorname{tp}(X)=\omega_{1}$, such that a $Y \in V$ with $Y \geqslant X, \operatorname{tp}(Y)<\alpha_{1}$, does not exist.

Proof. The appropriate notion of forcing will be $P_{\omega_{2}}$ where $P_{\alpha}\left(\alpha \leqslant \omega_{2}\right)$ is defined by induction. $P_{0}$ is just the trivial notion of forcing. $P_{\alpha+1}=P_{\alpha} * Q_{\alpha}$, where $Q_{\alpha}$ will be defined later; for limit $\alpha$ we take inverse limits when $\operatorname{cf}(\alpha)=\omega$ and direct limits if $\operatorname{cf}(\alpha)>\omega$.

We define $Q_{\alpha}$ (inside $\left.V^{P_{\alpha}}\right)$ as follows: $(f, A) \in Q_{\alpha}$ if and only if

(i) $\operatorname{Dom}(f) \in \omega_{1}$,

(ii) $\omega_{1}^{\alpha+1} \xi \leqslant f(\xi)<\omega_{1}^{\alpha+1}(\xi+1)(\xi \in \operatorname{Dom}(f))$, and

(iii) $\operatorname{tp}\left(A \cap\left[\omega_{1}^{\alpha+1} \xi, \omega_{1}^{\alpha+1}(\xi+1)\right]\right)<\omega_{1}^{\alpha+1}$.

The partial order is defined by $\left(f^{\prime}, A^{\prime}\right) \leqslant(f, A)$ iff $f^{\prime} \supseteq f, A^{\prime} \supseteq A$, and $f^{\prime}(\xi) \notin A$ for $\xi \in \operatorname{Dom}\left(f^{\prime}\right)-\operatorname{Dom}(f)$. It is easy to see that this relation is transitive.

In order to prove that forcing with any of the $P_{\alpha}\left(\alpha \leqslant \omega_{2}\right)$ cardinals and cardinal arithmetic remain we need some tools elaborated by Baumgartner [2]. A partial order $P$ is $\sigma$-closed if every decreasing sequence $p_{0} \geqslant p_{1} \geqslant \cdots \geqslant p_{n} \geqslant \cdots(n<\omega)$ has a lower bound. $P$ is well-met iff every two compatible elements have a greatest lower bound. $P$ is $\omega_{1}$-linked iff it can be written as $\cup\left\{R_{\tau}: \tau<\omega_{1}\right\}$ where the elements in any of the $R_{\tau}$ 's are pairwise compatible. Baumgartner proves that if $P_{\alpha}\left(\alpha \leqslant \omega_{2}\right)$ is defined as above and $Q_{\alpha}$ is (in $\left.V^{P_{\alpha}}\right) \sigma$-closed, well-met and $\omega_{1}$-linked, then $P_{\omega_{2}}$ is $\sigma$-closed and has the $\omega_{2}$-chain condition.

LEMMA 1. The partial ordering $Q_{\alpha}$ is $\sigma$-closed, well-met and $\omega_{1}$-linked.

Received by the editors November 30, 1982.

1980 Mathematics Subject Classification. Primary 03E35, 03E45, 04A99.

Key words and phrases. Covering lemma, inner models, iterated forcing.

(C)1983 American Mathematical Society $0002-9939 / 83 / 0000-1482 / \$ 01.50$ 
Proof. Assume $\left(f_{n}, A_{n}\right)$ is decreasing. At least, $f=\cup f_{n}, A=\cup A_{n}$ seems to give a candidate. As $\omega_{1}^{\alpha+1}$ is indecomposable into countably many smaller ordinals, the $(f, A)$ is a condition. Assume $\xi \in \operatorname{Dom}(f)-\operatorname{Dom}\left(f_{n}\right)$, and there is an $m$ with $\xi \in \operatorname{Dom}\left(f_{m}+1\right)-\operatorname{Dom}\left(f_{m}\right)$. Then $\xi \notin A_{m} \supseteq A_{n}$.

The ordering is $\omega_{1}$-linked as $(f, A)$ and $(f, B)$ are always compatible and by $\mathrm{CH}$ there are only $\omega_{1}$ first coordinates.

To prove that $Q_{\alpha}$ is well-met assume $(f, A)$ and $(g, B)$ are compatible and $\operatorname{Dom}(f)<\operatorname{Dom}(g)$. As some $(h, C) \leqslant(f, A),(g, B)$, so if $\xi \in \operatorname{Dom}(f)-\operatorname{Dom}(g)$ surely $\xi \in \operatorname{Dom}(h)-\operatorname{Dom}(f)$ so $g(\xi)=h(\xi) \notin A$. This gives that $(f \cup g, A \cup B)$ is the g.l.b. for $(f, A),(g, B)$.

We finish the proof by showing that the major conclusion of the theorem holds in $V^{P}$.

LEMMA 2. Forcing with $Q_{\alpha}$ defines a set $X_{\alpha}$ of order-type $\omega_{1}$ which cannot be covered by a set of order-type $\leqslant \omega_{1}^{\alpha+1}$ in the ground model.

Proof. Let $G$ be a generic set over $Q_{\alpha}$. Put $X_{\alpha}=\cup\{\operatorname{Rug}(f):(f, A) \in G\}$. As $D_{\xi}=\{(f, A): \xi \in \operatorname{Dom}(f)\}$ is clearly dense for $\xi<\omega_{1}$, the order-type of $X_{\alpha}$ is $\omega_{1}$. Assume $Y$ is the ground model $Y \subseteq \omega_{1}^{\alpha+2}$, tp $Y \leqslant \omega_{1}^{\alpha}$ and $(f, A) \Vdash \underline{X}_{\alpha} \subseteq Y$. Then ( $f, A \cup Y$ ) is a condition forcing $\underline{X}_{\alpha} \cap Y \subseteq \operatorname{Rug}(f)$ which is countable, so $\underline{X}_{\alpha} \subseteq Y$ cannot hold.

\section{REFERENCES}

1. K. Devlin and R. B. Jensen, Marginalia to a theorem of Silver, Logic Conference, Kiel 1974, Lecture Notes in Math., vol. 499, Springer-Verlag, Berlin, 1975, pp. 115-142.

2. J. E. Baumgartner, Iterated forcing, Proc. Cambridge Summer School on Set Theory, 1978 (to appear).

Department of Mathematics and Statistics, University of Calgary, Calgary, Alberta T2N IN4, CANADA

Current address: Department of Algebra and Number Theory, L. Eötvös University at Budapest, 1445 P.O.B. 323, Hungary 